\title{
Estimation of Carrier Frequency Offset for Generalized MC-CDMA Systems by Exploiting Hidden Pilots
}

\author{
Yi Ma, Member, IEEE, and Rahim Tafazolli, Member, IEEE
}

\begin{abstract}
This letter proposes a novel carrier frequency offset (CFO) estimation method for generalized multicarrier code-division multiple access systems in unknown frequency-selective channels utilizing hidden pilots. It is established that CFO is identifiable in the frequency domain by employing cyclic statistics (CS) and linear regression (LR) algorithms. We show that the CS-based estimator is capable of mitigating the normalized CFO (NCFO) to a small error value. Then, the LR-based estimator can be employed to offer more accurate estimation by removing the residual quantization error after the CS-based estimator. Simulation results are presented together with the theoretical analysis, and a good match between them is observed.
\end{abstract}

Index Terms-Carrier frequency offset (CFO), cyclic statistics (CS), hidden pilot, linear regression (LR), multicarrier code-division multiple access (MC-CDMA).

\section{INTRODUCTION}

G ENERALIZED multicarrier code-division multiple access (GMC-CDMA) has recently received increasing interests for high data rate transmissions [1], [2]. Relying on the precoding redundancy, [1] has shown that signal recovery for GMC-CDMA systems is independent of channel null (deep fade) locations. Hence, for uncoded systems, GMC-CDMA outperforms the conventional MC-CDMA in frequency-selective fading channels. As an orthogonal frequency-division multiplexing (OFDM)-based technique, GMC-CDMA is very sensitive to carrier frequency offset (CFO) [3]. A good CFO estimator is acquired to improve the overall system performance. In literature, many data-aided and nondata-aided CFO estimators have been reported for multicarrier systems (e.g., [6]-[10]). However, very few publications so far have addressed exploiting hidden pilots for the CFO estimation. This motivated us to develop a novel CFO estimator by using hidden pilots.

Originally, techniques using hidden pilots were proposed for low-complexity estimation of static channels (or slowly timevarying channels) in single-carrier systems (see [4] and [5]). In this letter, we deploy the hidden pilots in the GMC-CDMA system for the accurate CFO estimation. The proposed CFO estimator consists of two subestimators operating in the frequency domain. Using the cyclic-statistics (CS) algorithm, the first subestimator is able to mitigate the normalized $\mathrm{CFO}$ (NCFO) to a small error

Manuscript received April 21, 2005; revised May 26, 2005. This work was supported by EU-IST 4More, no. IST-2002-507039. The associate editor coordinating the review of this manuscript and approving it for publication was Dr. Geert Leus.

The authors are with Center for Communication Systems Research, University of Surrey, Surrey GU1 7XH, U.K. (e-mail: y.ma@surrey.ac.uk; r.tafazolli@surrey.ac.uk).

Digital Object Identifier 10.1109/LSP.2005.856883 value. This error is mainly from the quantization noise in the CS-based subestimator. The second subestimator is based on the linear-regression (LR) algorithm. It is shown that the LR-based estimator performs well for the case of small NCFO and is a good compensation to the CS-based estimator. Then, we use computer simulation to compare the proposed estimators with the pulse-shaping [8], CP-based [7], and null-subcarriers-based [9] approaches. Simulation results show that the proposed approach outperforms the others.

\section{GMC-CDMA WITH HIDDEN PILOTS}

The discrete-time equivalent model for the downlink of GMCCDMA system has been presented in [1] and [2]. Prior to transmission, the information-bearing symbols are grouped into blocks $\mathbf{s}_{M}(n)$ with the size of $M \times 1$, where $n$ denotes the block index. Then, these blocks are fed to a $K \times M$ block precoder $\Theta$ to produce blocks $\mathbf{s}_{K}(n)=\boldsymbol{\Theta} \mathbf{s}_{M}(n)$. In the GMC-CDMA system, $\boldsymbol{\Theta}$ is used for the user signatures and also introduces the precoding redundancy $(K>M)$ [1]. Letting $L_{t}=K-M$, we construct another block precoder $\Theta_{t}$ with the size of $K \times L_{t}$. This precoder is used to precode the hidden-pilot blocks $\mathbf{p}(n)$, with the input-output relationship of $\tilde{\mathbf{p}}(n)=\boldsymbol{\Theta}_{t} \mathbf{p}(n)$. Then, we feed the sum of $\mathbf{s}_{K}(n)$ and $\tilde{\mathbf{p}}(n)$ to the CP-OFDM modulator $\mathbf{F}$. The ma$\operatorname{trix} \mathbf{F}=\left[\mathbf{F}_{\mathrm{cp}}^{T}, \mathbf{F}_{K}^{T}\right]^{T}$ has the size of $J \times K\left(J=K+L_{\mathrm{cp}}\right)$, where $L_{\mathrm{cp}}$ denotes the length of cyclic prefix (CP), $(\cdot)^{T}$ denotes the matrix transpose, $\mathbf{F}_{K}$ denotes the inverse discrete Fourier transform (IDFT) matrix with the size of $K \times K$, and $\mathbf{F}_{\mathrm{cp}}$ is formed by collecting the last $L_{\mathrm{cp}}$ rows of $\mathbf{F}_{K}$. The output of CP-OFDM modulator (or transmitted block) is given by

$$
\mathbf{x}(n)=\mathbf{F W}\left[\mathbf{s}_{M}^{T}(n), \mathbf{p}^{T}(n)\right]^{T}
$$

where $\mathbf{W}=\left[\boldsymbol{\Theta}, \boldsymbol{\Theta}_{t}\right]$ is usually formed by a Walsh matrix in GMC-CDMA systems. The detail description on how to obtain the output (1) can be found in [1].

The transmitted blocks experience the propagation channel $\mathbf{h}=\left[h(0), h(1), \ldots, h\left(L_{u}\right)\right]^{T}$, where $L_{u}$ denotes the upper bound of the channel order. Considering the CFO, the received blocks can be formulated as (see [1] and [6])

$$
\mathbf{y}_{J}(n)=\boldsymbol{\Omega}_{J} \mathbf{H}_{l} \mathbf{x}(n)+\underbrace{\boldsymbol{\Omega}_{J} \mathbf{H}_{u} \mathbf{x}(n-1)}_{\mathrm{IBI}}+\mathbf{v}_{J}(n)
$$

where $\mathbf{H}_{l}$ and $\mathbf{H}_{u}$ are $J \times J$ lower and upper triangular matrices with entries $\left[\mathbf{H}_{l}\right]_{n, k}=h(n-k)$ and $\left[\mathbf{H}_{u}\right]_{n, k}=h(J+n-k) \cdot \mathbf{v}_{J}(n)$ is the zero-mean Gaussian noise with the size of $J \times 1$. The diagonal ma$\operatorname{trix} \Omega_{J}=\operatorname{diag}\left\{\Phi^{n J}, \Phi^{n J+1}, \ldots, \Phi^{(n+1) J-1}\right\}$ comprises the CFO information with $\Phi=\exp (j(2 \pi / K) \phi)$, where $\phi$ denotes 
the CFO normalized by the subcarrier spacing $\Delta f$. Normally, for the CP length $L_{\mathrm{cp}} \geq L_{u}$, the interblock interference (IBI) part can be removed by discarding $\mathrm{CP}$ at the receiver, and the remaining part becomes

$$
\mathbf{y}_{K}(n)=\Omega_{K} \mathbf{C}_{K} \mathbf{F}_{K} \mathbf{W}\left[\mathbf{s}_{M}^{T}(n), \mathbf{p}^{T}(n)\right]^{T}+\mathbf{v}_{K}(n)
$$

where $\boldsymbol{\Omega}_{K}=\operatorname{diag}\left\{\Phi^{n J+L_{\mathrm{cp}}}, \Phi^{n J+L_{\mathrm{cp}}+1}, \ldots, \Phi^{(n+1) J-1}\right\}$, $\mathbf{C}_{K}$ is the circulant channel matrix addressed in [1], and $\mathbf{v}_{K}(n)$ is the corresponding noise vector. We perform the DFT on $\mathbf{y}_{K}(n)$ and obtain the frequency-domain blocks as

$$
\begin{aligned}
\tilde{\mathbf{y}}_{K}(n)=\Phi^{n J+L_{\mathrm{cp}}}\left(\mathcal{D}_{K} \mathbf{W}[\right. & \left.\mathbf{s}_{M}^{T}(n), \mathbf{p}^{T}(n)\right]^{T} \\
& \left.+\mathbf{i}_{\mathbf{s}}(n)+\mathbf{i}_{\mathbf{p}}(n)\right)+\tilde{\mathbf{v}}_{K}(n)
\end{aligned}
$$

where $\tilde{\mathbf{v}}_{K}(n)=\mathbf{F}_{K}^{-1} \mathbf{v}_{K}(n), \mathbf{i}_{\mathbf{s}}(n)$, and $\mathbf{i}_{\mathbf{p}}(n)$ are the intercarrier interferences (ICIs) contributed by the information symbols and pilots, respectively; $\mathcal{D}_{K}=\mathbf{D}(\tilde{\mathbf{h}})$, where $\tilde{\mathbf{h}}$ denotes the channel frequency-response (CFR) with the size of $K \times 1$, and $\mathbf{D}(\mathbf{a})$ denotes the diagonal matrix with $\mathbf{a}$ in its diagonal. Then, we have the following three comments on (4).

1) The pilot blocks are assumed to be invariant with respect to the block index $n$. So, we can omit the index $n$ in pilotrelated terms $\tilde{\mathbf{p}}(n), \mathbf{p}(n)$ and $\mathbf{i}_{\mathbf{p}}(n)$ for convenience.

2) The ICI term $\mathbf{i}_{\mathbf{s}}(n)$ is contributed by $\mathbf{s}(n)$ and, thus, has the zero mean and is uncorrelated for the block index $n$ [3].

3) When $\phi$ and $\mathbf{h}$ are known at the receiver, the CFO and pilot blocks can be eliminated from (3). Then, the zero-forcing (ZF) equalization can be employed in the frequency domain. Reference [1] has shown that $\mathcal{D}_{K}$ has at most $L_{u}$ zeros on its diagonal, so the signal recovery is independent of channel null (deep fade) locations only when $L_{t} \geq L_{u}$ and any $M$ rows of $\Theta$ are linear independent.

Later on, we present how to use hidden pilots for the CFO estimation.

\section{ESTIMATION OF CFO}

\section{A. CS-Based Estimator}

Consider the autocorrelation of the received blocks (4) as

$$
\mathcal{C}_{\tilde{\mathbf{y}}_{K} \tilde{\mathbf{y}}_{K}}\left(n, n_{\tau}\right)=\mathcal{E}\left\{\tilde{\mathbf{y}}_{K}\left(n,+n_{\tau}\right) \tilde{\mathbf{y}}_{K}^{\mathcal{H}}(n)\right\}
$$

Based on the comments 1) and 2) in Section II, it is easy to obtain

where

$$
\mathcal{C}_{\tilde{\mathbf{y}}_{K} \tilde{\mathbf{y}}_{K}}\left(n_{\tau} \neq 0\right)=\Phi^{n_{\tau} J} \boldsymbol{\Psi}_{0}
$$

$$
\Psi_{0}=\mathcal{D}_{K} \mathcal{C}_{\tilde{\mathbf{p}} \tilde{\mathrm{p}}} \mathcal{D}_{K}^{\mathcal{H}}+\mathcal{D}_{K} \mathcal{C}_{\tilde{\mathrm{p}} \mathbf{i}_{\mathrm{p}}}+\mathcal{C}_{\tilde{\mathbf{p}} \mathbf{i}_{\mathrm{p}}}^{\mathcal{H}} \mathcal{D}_{K}^{\mathcal{H}}+\mathcal{C}_{\mathbf{i}_{\mathrm{p}} \mathbf{i}_{\mathrm{p}}}
$$

We can see that the matrix $\boldsymbol{\Psi}_{0}$ is constant with respect to the lag $n_{\tau}$. Therefore, $\mathcal{C}_{\tilde{\mathbf{y}}_{K} \tilde{\mathbf{y}}_{K}}\left(n_{\tau}>0\right)$ is a periodic function of $n_{\tau}$ with the period of $(K / J \phi)$ by assuming that the matrix $\boldsymbol{\Psi}_{0}$ is not zero (justification of this assumption will be given later). For $n_{\tau} \in(0, N-1]$, we perform the DFT on (6) and obtain (see [11] for DFT property)

$$
\begin{aligned}
\tilde{\mathcal{C}}_{\tilde{\mathbf{y}}_{K} \tilde{\mathbf{y}}_{K}}\left(m_{\tau}\right) & =\frac{1}{N-1} \sum_{n_{\tau}=1}^{N-1} \mathcal{C}_{\tilde{\mathbf{y}}_{K} \tilde{\mathbf{y}}_{K}}\left(n_{\tau}\right) e^{-j \frac{2 \pi}{N-1} m_{\tau} n_{\tau}} \\
& =e^{-j \frac{2 \pi}{N-1}\left(m_{\tau}-\frac{(N-1) J \phi}{K}\right)} \mathbf{\Psi}_{0} \mathcal{S}\left(m_{\tau}-\frac{(N-1) J \phi}{K}\right)
\end{aligned}
$$

where

$$
\mathcal{S}(x)=\frac{\sin (\pi x)}{(N-1) \sin \left(\frac{\pi}{N-1} x\right)} e^{-j \frac{N-2}{N-1} \pi x} .
$$

For $m_{\tau} \geq 0$, the norm of $\tilde{\mathcal{C}}_{\tilde{\mathbf{y}}_{K} \tilde{\mathbf{y}}_{K}}\left(m_{\tau}\right)$ is given by

$$
\left\|\tilde{\boldsymbol{C}}_{\tilde{\mathbf{y}}_{K} \tilde{\mathbf{y}}_{K}}\left(m_{\tau}\right)\right\|=\left\|\boldsymbol{\Psi}_{0}\right\|\left|\mathcal{S}\left(m_{\tau}-\frac{(N-1) J \phi}{K}\right)\right|
$$

which achieves its maximum along the $m_{\tau}$ direction only when $\left|\mathcal{S}\left(m_{\tau}-((N-1) J \phi / K)\right)\right|$ reaches its maximum, i.e., $m_{\tau}-$ $((N-1) J \phi / K)=0$. Since $m_{\tau}$ is an integer, the maximum of $\left|\mathcal{S}\left(m_{\tau}-((N-1) J \phi / K)\right)\right|$ should be located at

$$
\hat{m}_{\tau}=\left\lfloor\frac{(N-1) J \phi}{K}+\frac{1}{2}\right\rfloor
$$

where $\lfloor\cdot\rfloor$ denotes the integer floor. Hence, we can first determine such a $\hat{m}_{\tau}$ by searching the maximum of $\left\|\tilde{\boldsymbol{C}}_{\tilde{\mathbf{y}}_{K} \tilde{\mathbf{y}}_{K}}\left(m_{\tau}\right)\right\|$ over $m_{\tau} \in[0, N-2]$, and then, estimate the NCFO via

$$
\hat{\phi}=\frac{K \hat{m}_{\tau}}{(N-1) J} \text {. }
$$

Then, we provide the following three comments.

1) Estimation Range: For $m_{\tau} \in[0, N-2]$, the maximum identifiable NCFO, denoted by $\hat{\phi}_{\max }$, can be derived from (11)

$$
\hat{\phi}_{\max }=\frac{(N-2) K}{(N-1) J} .
$$

Due to the effect of frequency fold, the estimate range of CFO is given by $|\phi| \leq\lfloor((N-2) K / 2(N-1) J)\rfloor$.

2) Quantization Noise: Since $m_{\tau}$ is an integer, the relationship between $\phi$ and $m_{\tau}$ can be derived from (10) and (11) as

$$
\phi=\frac{K\left(m_{\tau}+\delta_{M}\right)}{(N-1) J}
$$

where $\left|\delta_{M}\right| \leq 0.5$ is the quantization noise. This noise may incur the CFO estimation error as

$$
|\hat{\phi}-\phi|=\frac{K\left|\delta_{M}\right|}{(N-1) J} \leq \frac{K}{2(N-1) J} .
$$

Certainly, this error can be further mitigated by increasing $N$.

3) Low-Complexity Approach: The computation complexity of the CS-based approach is mainly from the DFT operation and autocorrelation. Particularly, (8) needs the complexity of $\mathcal{O}\left(K^{2}(N-1) \log (N-1)\right)$ for the fast Fourier transform (FFT) operation. The low-complexity approach is intended to reduce the computation cost of FFT by considering the trace of $\mathcal{C}_{\tilde{\mathbf{y}}_{K} \tilde{\mathbf{y}}_{K}}\left(n_{\tau} \neq 0\right)$ as

$$
\operatorname{Tr}\left\{\mathcal{C}_{\tilde{\mathbf{y}}_{K} \tilde{\mathbf{y}}_{K}}\left(n_{\tau} \neq 0\right)\right\}=\Phi^{n_{\tau} J} \operatorname{Tr}\left\{\boldsymbol{\Psi}_{0}\right\}
$$

where $\operatorname{Tr}\{\mathbf{A}\}$ denotes the trace of $\mathbf{A}$. We find that $\boldsymbol{\Psi}_{0}$ is a Hermitian matrix, and thus, $\operatorname{Tr}\left\{\boldsymbol{\Psi}_{0}\right\}>0$. Then, the DFT of (15) is given by

$$
\begin{array}{r}
\operatorname{Tr}\left\{\tilde{\boldsymbol{C}}_{\tilde{\mathbf{y}}_{K} \tilde{\mathbf{y}}_{K}}\left(m_{\tau}\right)\right\}=e^{-j \frac{2 \pi}{N-1}\left(m_{\tau}-\frac{(N-1) J \phi}{K}\right)} \operatorname{Tr}\left\{\boldsymbol{\Psi}_{0}\right\} \\
\cdot \mathcal{S}\left(m_{\tau}-\frac{(N-1) J \phi}{K}\right) .
\end{array}
$$

For $m_{\tau} \geq 0$, the absolute value of $\operatorname{Tr}\left\{\tilde{\mathcal{C}}_{\tilde{\mathbf{y}}_{K} \tilde{\mathbf{y}}_{K}}\left(m_{\tau}\right)\right\}$ is given by

$$
\left|\operatorname{Tr}\left\{\tilde{\mathcal{C}}_{\tilde{\mathbf{y}}_{K} \tilde{\mathbf{y}}_{K}}\left(m_{\tau}\right)\right\}\right|=\operatorname{Tr}\left\{\boldsymbol{\Psi}_{0}\right\}\left|\mathcal{S}\left(m_{\tau}-\frac{(N-1) J \phi}{K}\right)\right| .
$$

Then, the NCFO can be estimated by searching the maximum of $\left|\operatorname{Tr}\left\{\tilde{\mathcal{C}}_{\tilde{\mathbf{y}}_{K} \tilde{\mathbf{y}}_{K}}\left(m_{\tau}\right)\right\}\right|$. The trace-based algorithm only needs one 
FFT and, thus, reduces the computation cost of FFT to $\mathcal{O}((N-$ 1) $\log (N-1))$.

\section{B. LR-Based Estimator}

The linear regression estimator is based on the following equation:

$$
\mathbf{C}\left(n_{\tau}\right)=\mathcal{C}_{\tilde{\mathbf{y}}_{K} \tilde{\mathbf{y}}_{K}}\left(n_{\tau}\right) \mathcal{C}_{\tilde{\mathbf{y}}_{K} \tilde{\mathbf{y}}_{K}}^{\mathcal{H}}(1)=\Phi^{\left(n_{\tau}-1\right) J} \boldsymbol{\Gamma}
$$

where $\boldsymbol{\Gamma}=\mathbf{\Psi}_{0} \boldsymbol{\Psi}_{0}^{\mathcal{H}}$ is a Hermitian matrix. The trace of $\mathbf{C}\left(n_{\tau}\right)$ is given by

$$
\operatorname{Tr}\left\{\mathbf{C}\left(n_{\tau}\right)\right\}=\Phi^{\left(n_{\tau}-1\right) J} \operatorname{Tr}\{\boldsymbol{\Gamma}\} .
$$

Due to $\boldsymbol{\Psi}_{0} \neq \mathbf{0}, \operatorname{Tr}\{\boldsymbol{\Gamma}\}$ should be positive. So, we can obtain the phase of $\operatorname{Tr}\left\{\mathbf{C}\left(n_{\tau}\right)\right\}$ using

$$
\begin{aligned}
\varphi\left(n_{\tau}\right) & =\arg \left\{\operatorname{Tr}\left\{\mathbf{C}\left(n_{\tau}\right)\right\}\right\} \\
& =\frac{2 \pi J \phi}{K}\left(n_{\tau}-1\right)-2 \pi\left\lfloor\frac{2 n_{\tau}+T_{\varphi}}{2 T_{\varphi}}\right\rfloor
\end{aligned}
$$

where $\arg \{x\}$ is a function to obtain the phase of $x$, for $\arg \{x\} \in[-\pi, \pi), T_{\varphi}=(K / J \phi)$ is the period of the function $\varphi\left(n_{\tau}\right)$. Assuming $T_{\varphi}$ to be known, we can form a first-order polynomial $f\left(n_{\tau}\right)=a n_{\tau}+b$ and determine the parameters $(a, b)$ by solving the following equation:

$$
(\hat{a}, \hat{b})=\arg \min _{(\hat{a}, \hat{b})} \sum_{n_{\tau}=1}^{\left\lfloor T_{\varphi} / 2\right\rfloor}\left|\varphi\left(n_{\tau}\right)-f\left(n_{\tau}\right)\right|^{2} .
$$

It is easy to find that $\hat{a}$ is the estimated slope of $\varphi\left(n_{\tau}\right)$, which is identifiable by using the LR algorithm. Then, the NCFO $\phi$ can be determined by

$$
\hat{\phi}=\frac{K}{2 \pi J} \hat{a} .
$$

The following points should be noted for the LR-based approach.

1) Estimation Performance: Equation (21) shows that the curve fitting performance improves with the increase of $T_{\varphi}$. However, $T_{\varphi}$ is inverse proportional to the NCFO. Hence, the estimation performance becomes worse with the increase of NCFO.

2) About $\left\lfloor T_{\varphi} / 2\right\rfloor$ : Equation (21) shows that the knowledge of $\left\lfloor T_{\varphi} / 2\right\rfloor$ is required for successful CFO estimation. To determine $\left\lfloor T_{\varphi} / 2\right\rfloor$, we perform the differential operation on $\varphi\left(n_{\tau}\right)$ and have

$$
\begin{aligned}
\Delta \varphi\left(n_{\tau}\right) & =\varphi\left(n_{\tau}+1\right)-\varphi\left(n_{\tau}\right) \\
& = \begin{cases}\gamma, & \text { for } n_{\tau}=\left\lfloor(2 i+1) T_{\varphi} / 2\right\rfloor \\
\frac{2 \pi J \phi}{K}, & \text { otherwise }\end{cases}
\end{aligned}
$$

where $i=0,1, \ldots$, and $\gamma$ is very close or equal to $-2 \pi .{ }^{1}$ Due to $|(2 \pi J \phi / K)| \ll|\gamma|$, we can find $\left\lfloor T_{\varphi} / 2\right\rfloor$ by searching the first location of $\gamma$ over $n_{\tau} \in(0, N-1)$. If they does not have such a $\gamma$, then we set $\left\lfloor T_{\varphi} / 2\right\rfloor=N-1$.

\section{About $\mathbf{\Psi}_{0}$}

In order to guarantee the CFO identifiability, we need the condition $\boldsymbol{\Psi}_{0} \neq \mathbf{0}$. Because $\tilde{\mathbf{p}}$ and $\mathbf{i}_{\mathbf{p}}$ are constant, (7) can be rewritten into

$$
\boldsymbol{\Psi}_{0}=\mathcal{D}_{K} \tilde{\mathbf{p}} \tilde{\mathbf{p}}^{\mathcal{H}} \mathcal{D}_{K}^{\mathcal{H}}+\mathcal{D}_{K} \tilde{\mathbf{p}}_{\mathbf{p}}^{\mathcal{H}}+\mathbf{i}_{\mathbf{p}} \tilde{\mathbf{p}}^{\mathcal{H}} \mathcal{D}_{K}^{\mathcal{H}}+\mathbf{i}_{\mathbf{p}} \mathbf{i}_{\mathbf{p}}^{\mathcal{H}}
$$

In order to assure $\boldsymbol{\Psi}_{0} \neq \mathbf{0}$, the pilot design should fulfill $\mathcal{D}_{K} \tilde{\mathbf{p}}=\mathbf{0}$. Since $\mathcal{D}_{K}$ contains at most $L_{u}$ zeros on its diag-

${ }^{1} \gamma=-2 \pi$ holds only when $T_{\varphi}$ is an integer. onal, this condition can be achieved when $\tilde{\mathbf{p}}$ has at least $L_{u}+1$ nonzero elements.

\section{Simulation Results AND ANALYsis}

The root-mean-square error (RMSE) $\sqrt{1 / \mathcal{I} \sum_{i=0}^{\mathcal{I}-1}\left|\hat{\phi}_{i}-\phi\right|^{2}}$ was used to benchmark the CFO estimation performance. Here, $\mathcal{I}$ is the number of Monte Carlo trials. The frequency-selective fading channel was modeled as a finite impulse response (FIR) filter with the maximum order of $L_{u}=4$. Each tap was randomly generated according to the Rayleigh distribution with the variance of $1 /\left(L_{u}+1\right)$. The block precoder $\mathbf{W}$ was formed by the $K \times K$ Walsh matrix. The parameters of GMC-CDMA systems were given by $K=32, M=28, L_{\mathrm{cp}}=4, L_{t}=4$. The information-bearing symbols were drawn from the quadrature phase-shift keying (QPSK) constellation with the equal probability. The subcarrier spacing $\Delta f$ of $312.5 \mathrm{kHz}$ was the same as HIPERLAN/2 [13]. Elements in the pilot block $\mathbf{p}$ were identical. The pilot-to-information power ratio was given by $L_{t} / M$. The signal-to-noise ratio (SNR) is defined by the average received symbol energy to noise $E_{s} / \mathcal{N}_{o}$ [14]. Based on the above parameters, we find that the block $\tilde{\mathbf{p}}$ results in eight nonzero elements with equal amplitudes. As shown in Section III-C, the CFO identifiability can be guaranteed by employing this kind of pilot design.

Test Case 1: This experiment examines the estimation performance as a function of NCFO with SNR $=5$ and 15 $\mathrm{dB}$, respectively. The RMSE results were obtained by taking the average of $\mathcal{I}=500$ Monte Carlo trials. Each trial collected $N=200$ GMC-CDMA blocks for the CFO estimation. Fig. 1(a) shows that the RMSE for the CS-based approach is very stable (around $2 \times 10^{-3}$ ). This result has a good match with the quantization error in (14). In the range of large NCFO (e.g., $|\phi|>0.15$ ), we can observe that the LR-based approach cannot offer a good estimation due to the relatively short $T_{\varphi}$. The estimation performance improves significantly with the decrease of NCFO. When NCFO is small (e.g., $|\phi|<0.1$ ), the LR-based approach outperforms the CS-based approach.

Test Case 2: In order to indicate the performance-complexity tradeoff, we examine the RMSE results as a function of data record length (DRL) $N$ with $\phi=0.0625$ and the typical SNR = $12 \mathrm{~dB}$. Fig. 1(b) shows that RMSE for CFO estimators decreases rapidly with the increase of $N$. They can achieve the best performance offered by the pulse-shaping-based approach [8] only using $N=20$ blocks.

Test Case 3: To evaluate the subestimators for different SNR cases, we plot the RMSE results in Fig. 2 with the NCFO $\phi=0.0625$ and the DRL $N=100$ blocks.Fig. 2 shows that the CS-based subestimator is not sensitive to the SNR. The RMSE result for the LR-based estimator becomes stable and offers the best performance for SNR $>10 \mathrm{~dB}$. Interestingly, we observe that the low-complexity CS-based approach offers the same performance as the CS-based approach. In order to compare with state-of-the-art approaches, we also plot the RMSE results for the pulse-shaping, CP-based [7] and null-subcarriers (NS)-based [9] CFO estimators. Fig. 2 shows that the proposed estimators outperform the pulse-shaping-based and CP-based estimator. For the fair comparison with the NS-based approach, we investigate the OFDM system with the same setup as the GMC-CDMA system. The number of NSs is $N_{z}=4$, which is 


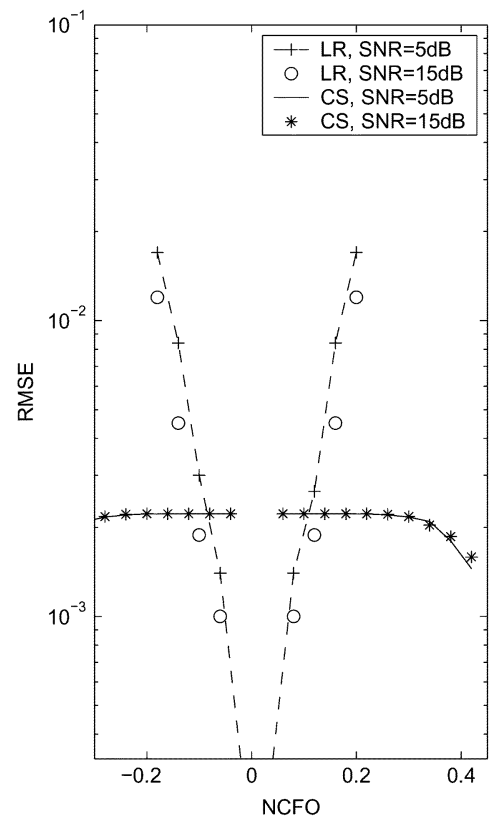

(a)

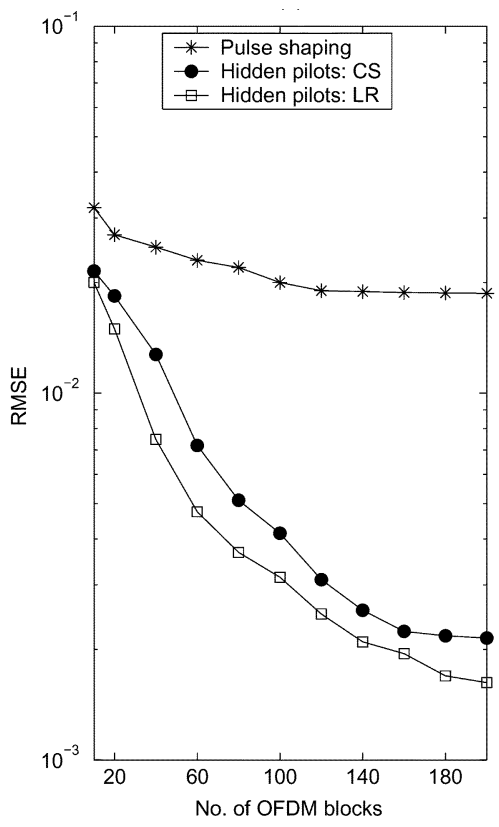

(b)

Fig. 1. RMSE results as a function of NCFO and DRL.

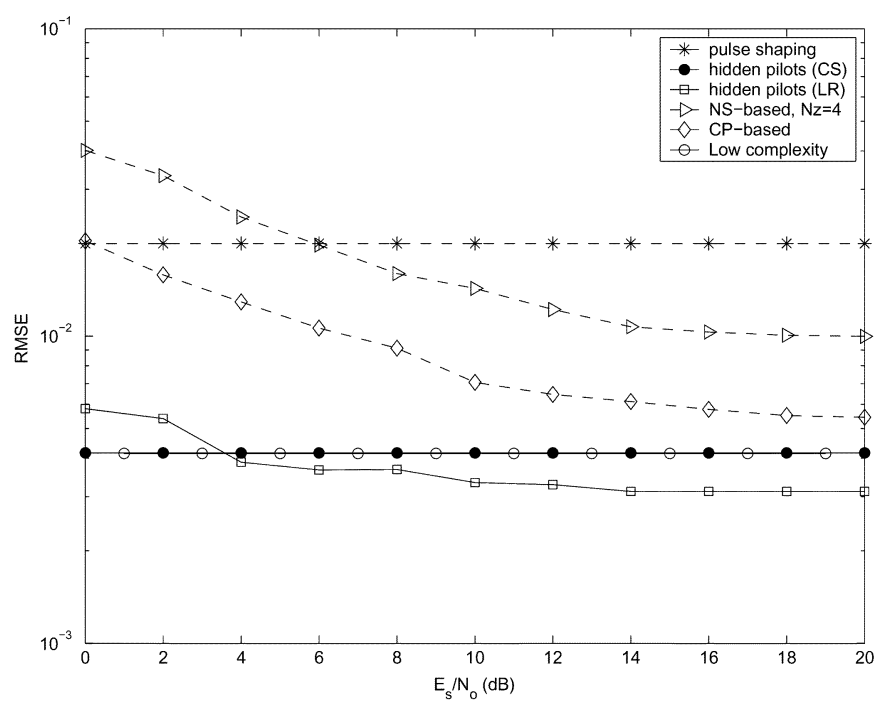

Fig. 2. RMSE results as a function of SNR.

the same as the number of hidden pilots in the GMC-CDMA system. Fig. 2 shows that the NS-based approach is sensitive to the SNR (particularly in the low SNR range). The proposed approaches also outperform the NS-based one.

\section{CONCLUSION}

Utilizing the hidden pilots, this letter has proposed a novel CFO estimators for GMC-CDMA in unknown frequency-selective channels. It has been established that the CFO is identifiable in the frequency domain by employing the CS-based and LR-based approaches. Both theoretical and simulation results have shown the excellent estimation performance of the proposed CFO estimator.

\section{REFERENCES}

[1] Z. Wang and G. B. Giannakis, "Wireless multicarrier communications: Where Fourier meets Shannon," IEEE Signal Process. Mag., vol. 17, no. 3, pp. 29-48, May 2000.

[2] G. B. Giannakis, Z. Wang, A. Scaglione, and S. Barbarossa, "AMOURgeneralized multicarrier transceiver for blind CDMA regardless of multipath," IEEE Trans. Commun., vol. 48, no. 12, pp. 2064-2076, Dec. 2000.

[3] T. Pollet, M. V. Bladel, and M. Moeneclaey, "BER sensitivity of OFDM systems to carrier frequency offset and Wiener phase noise," IEEE Trans. Commun., vol. 43, no. 2, pp. 191-193, Feb. 1995.

[4] F. Mazzenga, "Channel estimation and equalization for M-QAM transmission with a hidden pilot sequence," IEEE Trans. Broadcast., vol. 46, no. 2, pp. 170-176, Jun. 2000.

[5] J. K. Tugnait and W. Luo, "On channel estimation using superimposed training and first-order statistics," IEEE Commun. Lett., vol. 7, no. 9, pp. 413-415, Sep. 2003.

[6] J. Li, G. Liu, and G. B. Giannakis, "Carrier frequency offset estimation for OFDM-based WLAN," IEEE Signal Process. Lett., vol. 8, no. 3, pp. 80-82, Mar. 2001.

[7] J.-J. van de Beck et al., "ML estimation of timing and frequency offset in OFDM systems," IEEE Trans. Signal Process., vol. 45, no. 7, pp. 1800-1805, Jul. 1997.

[8] H. Bolcskei, "Blind estimation of symbol timing and carrier frequency offset in wireless OFDM systems," IEEE Trans. Commun., vol. 49, no. 6, pp. 988-999, Jun. 2001.

[9] H. Liu and U. Tureli, "A high efficiency carrier estimator for OFDM communications," IEEE Commun. Lett., vol. 2, no. 4, pp. 104-106, Apr. 1998.

[10] N. Lashkarian and S. Kiaei, "Class of cyclic-based estimators for frequency offset estimation of OFDM systems," IEEE Trans. Commun., vol. 48, no. 12, pp. 2139-2149, Dec. 2000.

[11] A. V. Oppenheim and R. W. Schafer, Discrete-Time Signal Processing. Englewood Cliffs, NJ: Prentice-Hall, 1989.

[12] Y. Zhao and S. G. Haggman, "Intercarrier interference self-cancellation scheme for OFDM mobile communication systems," IEEE Trans. Commun., vol. 49, no. 7, pp. 1185-1191, Jul. 2001.

[13] Broadband Radio Access Networks (BRAN); High Performance Radio Local Area Networks (HIPERLAN) Type 2: Physical Layer, Dec. 1999. ETSI, document DTS0 023003.

[14] J. Proakis, Digital Communications, 4th ed. New York: McGraw-Hill, 2000. 